\title{
INVERSIÓN EXTRANJERA DIRECTA Y LIBERTAD ECONÓMICA COMO DETERMINANTES DEL CRECIMIENTO ECONÓMICO DE ECUADOR EN EL CORTOY LARGO PLAZO
}

\section{FOREIGN DIRECT INVESTMENT AND ECONOMIC FREEDOM AS DETERMINANTS OF ECUADOR'S ECONOMIC GROWTH IN THE SHORT AND LONG TERM}

Fecha de recepción: 13 de noviembre de 2018.

Fecha de aceptación: 27 de diciembre de 2018.

Código JEL: C32, C51, E23, F43.

Código DOI: 10.25097/rep.n29.2019.06
Cristian Ortiz ${ }^{1}$

(cristian.ortiz@unl.edu.ec)

Andrea Salinas ${ }^{2}$

(andrea.salinas@unl.edu.ec)

Rafael Alvarado ${ }^{3}$

(rafaalvaradolopez@gmail.com)

Pablo Ponce ${ }^{4}$

(pablo_vpo@hotmail.com)

\section{Resumen}

La presente investigación examina el efecto de la inversión extranjera directa (IED) y la libertad económica (LE) en el crecimiento económico (PIB) en Ecuador. Se utilizó datos de series de tiempo del Banco Mundial y Heritage Fundation, correspondientes al periodo 1980-2017. A través de modelos ARDL y ECM, los resultados describen una relación estable a corto y largo plazo entre las variables. También encontramos causalidad bidireccional entre la formación bruta de capital fijo y la IED y causalidad unidireccional desde la IED hacia el PIB. Una implicación de política económica derivada de esta investigación es el mantener un buen ambiente macroeconómico y jurídico. Además de generar subsidios no solo para atraer capital extranjero, sino también subsidios educativos que podrían ayudar a los pobres a alcanzar la cantidad mínima de educación necesario para lograr generar encadenamientos productivos de la IED en las economías locales.

Palabras clave: Crecimiento económico, inversión extranjera directa, libertad económica.

\section{Abstract}

This research examines the effect of foreign direct investment (FDI) and economic freedom (LE) on economic growth (GDP) in Ecuador. World Bank and Heritage Foundation time series data for the 1980-2017 period are published. Through the ARDL and ECM models, the results of a stable short and long term relationship between the variables. We also find bidirectional causality between the formation of fixed capital and FDI and unidirectional causality from FDI to GDP. One implication of economic policy derived from this research is to maintain a good macroeconomic and legal environment. In addition, not only to attract foreign capital, but also to help the poor achieve the minimum amount of education necessary to achieve productive linkages of FDI in local services.

Keywords: Economic growth, foreign direct investment, economic freedom.

\footnotetext{
1 Carrera de Economía. Universidad Nacional de Loja. Loja, Ecuador. Orcid: 0000-0002-9395-7228

2 Carrera de Economía. Universidad Nacional de Loja. Loja, Ecuador.

3 Carrera de Economía. Universidad Nacional de Loja. Loja, Ecuador. Orcid: 0000-0002-3213-5431

4 Carrera de Economía, Universidad Nacional de Loja - Ecuador, ORCID: 0000-0003-2717-0176. Autor para correspondencia.
} 


\section{Introducción}

Según datos del World Development Indicators (WDI) del Banco Mundial (2017), los flujos globales de Inversión Extranjera Directa (IED) como porcentaje del PIB ascendieron de 0,5\% en 1970 a 2,5\% en 2017, aunque a lo largo del periodo histórico de datos se han experimentado porcentajes de participación más altos de la IED sobre el Producto Interno Bruto (PIB) Mundial, por ejemplo 4,3\% en el 2000 y 5,2\% en 2005, los datos indican que en los últimos años los flujos de IED se han concentrado de mayor manera en los países de ingresos bajos $(3,5 \%$ del PIB en 2017) en comparación con en el resto de grupos de países con distintos niveles de ingreso que considera el Banco Mundial, como son los países de ingreso alto, medio alto y medio bajo (2,6\%; $1,8 \%$ y $2,1 \%$ del PIB respectivamente en 2017$)$, y esta tendencia se ha mantenido aproximadamente los últimos cinco años. En el Ecuador, la participación de la IED sobre el producto no ha logrado mantener porcentajes importantes, ya que en los años 2015, 2016 y 2017 ha sido de 1,33\%; 0,76\% y 0,59\% del PIB respectivamente y en promedio desde 1980 a 2016 de $1,23 \%$ del PIB, porcentajes que son muy bajos si comparamos con los porcentajes de países como Perú y Bolivia, quienes reportan valores promedio en el mismo periodo de 2,76 y $3,56 \%$ del PIB respectivamente.

Aunque de manera teórica, la literatura menciona que los países con una mayor proporción de IED sobre la economía, tienen un efecto de convergencia sobre el crecimiento de la productividad y el crecimiento del PIB; existe evidencia que muestra que los flujos de IED solo son positivos en los países de ingresos altos y no significativos, y hasta negativos en los países de ingresos medios e ingresos medios-bajos como el caso de países de América Latina (Alvarado, Iñiguez y Ponce 2017), estos resultados pueden argumentarse dado la heterogeneidad de las estructuras económicas y encadenamientos productivos ineficientes de los países, que no generan crecimientos sólidos y sostenibles en el largo plazo. Autores como Bhagwati y Srinivasan (2002), relacionan la apertura al comercio internacional y el crecimiento, y explican el fracaso de las políticas comerciales proteccionistas llevadas a cabo en las décadas posteriores a la Segunda Guerra Mundial por parte de países en desarrollo. Existen argumentos que demuestran que los niveles de movilidad del capital, que a su vez son potenciados por la globalización, afectan el crecimiento de los países (Herranz, 2009); no obstante, Benassy-Quere et al. (2001) demostró que la volatilidad del tipo de cambio era perjudicial para la IED en los países en vías de desarrollo.

Según Sánchez (2010) la libertad económica en los últimos 14 años ha avanzado en casi todo el mundo. Las regiones con mayor libertad son: Norteamérica, Europa y Asía-Pacífico. En Norteamérica, los países considerados con un índice libertad mayor son las economías de Estados Unidos y Canadá; en Europa, las economías de Reino Unido, Dinamarca, Holanda y Alemania; mientras que en Asia-Pacífico son las economías de Hong Kong, Singapur, Australia y Japón. La región Sudamericana y Centroamericana es la de peor desempeño en materia de libertad a causa de los retrocesos experimentados en las economías de Venezuela, Cuba y Bolivia.

Chile es el país que cuenta con un índice de libertad económica mejor posicionado que el resto de las economías de la región. La región de África del Norte incrementa su índice global ligeramente, pero no lo suficiente debido a los obstáculos impuestos a la libertad económica en Marruecos e Irán (Sánchez, 2010). El grado de libertad económica será menor en el contexto de una economía con un alto nivel de inestabilidad político-institucional, por tanto, se debe promover una democracia estable que garantiza los derechos individuales a la propiedad y el cumplimiento de los contratos a través de las generaciones, tomando como ejemplo a las actuales economías desarrolladas.

En este contexto, Smith (1776), Friedman (1996) y Hayek (1960), los principales precursores de este pensamiento, enfatizan la libertad en ámbitos como el comercio y coordinación del 
mercado, que son el combustible para que haya progreso económico, sin el intercambio y la actividad empresarial coordinada a través de los mercados, los estándares de la vida moderna serían imposibles, y señalan que el papel del gobierno es el de conceder libertad económica, democracia, acceso a oportunidades en educación, servicios de salud y calidad de vida, así como, participar como un ente regulador en casos específicos, pues la economía se regula por la mano invisible de la oferta y la demanda.

La hipótesis central consiste en que, tanto la IED como la libertad económica (LE) tienen efectos positivos en el crecimiento económico. En este contexto, el principal objetivo de esta investigación es examinar la relación a corto y largo plazo entre el crecimiento económico con respecto a la inversión extranjera directa y libertad económica en el Ecuador, utilizando econometría de datos de series de tiempo, debido a la escaza evidencia empírica referente al tema, especialmente para Ecuador. Por tanto, en el proceso se realizó un análisis econométrico que muestra la relación entre la inversión extranjera directa y la libertad económica, y su aporte al crecimiento económico.

En esta investigación, se utiliza el índice de libertades civiles como proxi de la variable independiente libertad económica, que proporciona una idea de las características de un entorno propicio para la prosperidad. Finalmente, es importante acotar que la presente investigación contribuye a la escaza literatura referente al tema, además de demostrar la relación entre las variables por medio de modelos dinámicos ECM y ARDL y el test de causalidad de Engle y Granger (1987).

En este proceso, mediante el test de Dickey y Fuller aumentado (ADF, 1979), se identificó la estacionariedad de las series de tiempo, en donde los resultados demuestran que las variables son series estacionarias de orden I (1), luego se estimó modelos ARDL y ECM para observar si existe una relación dinámica a largo o corto plazo respectivamente, y finalmente se realizó la prueba de Engle y Granger (1987) con el propósito de verificar la existencia de causalidad entre las variables.

Este trabajo incluye cuatro secciones adicionales a la introducción. La sección dos proporciona un conciso resumen que sustenta el efecto de la inversión extranjera directa y la libertad económica con el crecimiento económico, incorporando una breve revisión de literatura empírica del tema. La sección tres describe los datos y plantea la estrategia econométrica. La discusión de resultados ocupa la sección cuatro. Finalmente, la quinta sección abarca conclusiones e implicaciones de política económica.

\section{Revisión de la literatura}

Existen varios autores que investigan los factores que intervienen en el desarrollo de un país, por ejemplo Tsai (1995) sugiere que el nivel de desarrollo económico producto de entradas de capital, es generalmente un determinante crucial de la distribución del ingreso, mientras que Tung y Cho (2001), sostienen que para que esto funcione es necesario contar con incentivos fiscales para atraer inversión, tal como ocurrió en la última década en países como China.

La evidencia empírica se divide en algunas secciones. Primero, se parte con investigaciones que involucran a la inversión extranjera directa y al crecimiento económico. Segundo, la influencia e importancia de la libertad económica. Tercero, investigaciones que analicen técnicas de causalidad entre las variables. Cuarto, investigaciones que incluyen al capital y trabajo determinantes del crecimiento y que son utilizadas como variables de control en el presente estudio.

La IED permite crear un vínculo duradero con fines económicos y empresariales a largo plazo entre dos países, así lo corroboran Gomes y Veiga (2013); Anwar y Nguyen (2010); Huang, 
Chen, Huang y Yang (2016); Anwar y Sun (2011), afirmando su importancia para los efectos de la globalización financiera y el aprovechamiento de reglamentaciones que fortalezcan la estructura económica (Adams y Opoku, 2015). La IED también puede favorecer como instrumento para aumentar el crecimiento económico, dado que puede actuar como un mecanismo para acumular capital físico y transferir capital humano al país receptor, lo que puede aumentar la tasa de crecimiento económico. De esta manera la transferencia de tecnología aumenta la eficiencia de los factores de producción y esto, a su vez reduce la brecha tecnológica entre empresas nacionales e internacionales (Anwar y Nguyen, 2010). Asimismo, la IED puede actuar como un vehículo de transferencia de tecnología entre países desarrollados y en desarrollo (Borensztein et al., 1998).

La IED se ha relacionado con el crecimiento económico en diversos países. Un estudio llevado a cabo por Almfraji y Almsafir (2014) indica que la relación entre la IED y el crecimiento económico es principalmente positivo, en otros casos es negativo o nulo. Esta variedad de efectos de la IED se debe a factores como capital humano, mercados financieros bien desarrollados, complementariedad entre la inversión nacional y la extranjera, los regímenes comerciales abiertos entre otros. Existe una amplia literatura teórica y empírica que favorece la IED como instrumento para aumentar el crecimiento económico, por ejemplo la evidencia que señala que economías con una mayor proporción de inversión extranjera directa tienen un mayor efecto de convergencia y crecimiento de la productividad y del PIB, y se argumenta que existe una correlación positiva entre la IED y el crecimiento económico promovido por la competitividad, un capital humano adecuado, estabilidad económica y mercados liberalizados (Bengoa y SanchezRobles, 2003; Oscar, Díaz y Navarrete, 2017), además de enfatizar la idiosincrasias del país y del período que son determinantes importantes en cuanto a la comprensión del crecimiento económico (Belaşcu, Popovici y Horobeț, 2009).

En esta misma línea, Chen y Zulkifli (2012) revela la existencia de una relación positiva en el largo plazo entre la IED y el crecimiento económico. Igualmente Nistor (2014), indica que los flujos de IED tienen un impacto positivo en el crecimiento económico de Rumania. Además, Fadhil y Almsafir (2015) mediante la prueba de cointegración de Johansen demuestran con resultados que la entrada de IED junto con el desarrollo del capital humano contribuye al crecimiento económico, por lo que sugiere que el desarrollo del capital humano es un atractivo para la entrada de la IED. En esta misma línea está Pegkas (2015), mediante su análisis empírico emplea métodos FMLOS y DOLS y confirma la existencia de una relación de cointegración positiva en el largo plazo entre el stock de IED y el crecimiento de la economía. Complementario a los resultados anteriores, lamsiraroj (2016) encuentra que los efectos de la IED están asociados positivamente con el crecimiento y viceversa, mientras que la fuerza laboral, la apertura comercial y la liberta económica son otros determinantes fundamentales de la IED, que a su vez estimula aún más el crecimiento económico.

Sin embargo, existen algunos resultados que contradicen el efecto positivo de la inversión extranjera directa sobre el crecimiento económico. Los resultados encontrados por Belloumi (2014) para Túnez van en contra de la idea generalizada de que la IED genera efectos positivos en el crecimiento económico, acompañada de la ausencia de una causalidad significativa de Granger de la IED hacia el crecimiento económico. En este mismo sentido, Sokhanvar (2019) encuentra, no solo una relación insignificante entre la IED y el crecimiento económico, sino que la IED tiene un impacto negativo en el crecimiento económico para 5 países de la UE. Esto podría asociarse a que los países necesitan mercados financieros bien desarrollados para aprovechar las externalidades positivas que genera la IED sobre la economía (Alfaro, Chanda, Kalemli-Ozcan y Sayek, 2004). Finalmente, Gunby, Jin y Robert Reed (2017) mediante un meta - análisis, encuentran que el efecto de la IED en el crecimiento de China es insignificante y este se debería a otros factores. Una conclusión similar exponen Alvarado, Iñiguez y Ponce (2017), quienes sugieren que la IED no es un mecanismo adecuado para acelerar el crecimiento 
económico en América Latina, con la excepción de los países de altos ingresos, pues sostienen que países de ingresos medianos y bajos de la región deberían promover instrumentos fiscales que permitan que la inversión a genere vínculos con el sector productivo local.

La segunda parte involucra a la libertad económica y su influencia sobre el crecimiento económico. Desde Adam Smith, los economistas han defendido la libertad para elegir y proveer recursos, argumentando que la competencia en los negocios, intercambios comerciales y la protección de los derechos de propiedad, son ingredientes fundamentales para el progreso económico (Sturm, 2001). De igual manera Azman-Saini, Baharumshah y Law (2010); Compton, Giedeman y Hoover (2014) afirman que esta variable es importante para el crecimiento a largo plazo, especialmente en países en vías de desarrollo, que en muchas ocasiones son dependientes de economías más fuertes. Tanto la cantidad de mano de obra, la apertura al comercio y la libertad económica son determinantes para generar un crecimiento económico (lamsiraroj, 2016; Bengoa y Sanchez-Robles, 2003). Tanto Dawson (2003) como Sturm (2001) advierten que tanto los niveles de libertad económica como los cambios en la misma, ejercen un impacto significativo sobre el crecimiento, llevando a los países a su nivel de crecimiento de estado estacionario de forma más rápida.

Dentro de este último enfoque también aparece el papel de una estructura macroeconómica sólida, que es atractiva para potenciales inversores, por tanto, la inversión no solo debe hacerse en capital físico sino también en capital humano, para aumentar la competitividad y repunte del país, por medio del aprovechamiento de los recursos dados en la nación. Los países que han mejorado su competitividad y han abierto sus sociedades a nuevas ideas, productos e innovaciones han logrado en gran medida los elevados niveles de progreso social que exigen sus ciudadanos (MIller y Kim, 2016), y mejores condiciones locales atraen a empresas extranjeras (Alfaro, Chanda, Kalemli-Ozcan y Sayek, 2004; Herrera-Echeverri, Haar y Estévez-Bretón, 2014).

En tercer lugar, análisis de causalidad sugieren que las relaciones entre estas variables puede producirse de manera unidireccional y bidireccional, por ejemplo Abdouli y Hammami (2017) ; Dritsaki y Stiakakis (2014), demuestran que existe causalidad unidireccional entre el crecimiento económico y la inversión extranjera directa.

Autores como Murphy y Topel (2016) indican que la inversión trae tecnología, nuevos conocimientos, administración altamente calificada e inversiones a empresas locales que confieren una ventaja competitiva y economías de escala aumentando así su productividad; de la misma manera lo menciona Hussain (2011), contrario a lo encontrado por Gunby, Jin y Reed (2017) quienes afirman que en algunos niveles de ingreso la causalidad es unidireccional desde el PIB hacia la IED y por lo tanto el fomento de la IED no siempre mejora la economía, incluso el trasplantar las políticas favorecen a unos países, probablemente tendrán poco o nulo impacto en otros, en el mismo contexto Szkorupová (2014) asevera que existen variables que poseen un mayor efecto en el PIB como por ejemplo las exportaciones. Por su parte, Abdouli y Hammami (2017); Dritsaki y Stiakakis (2014) contrastan estos resultados con la estimación de un modelo VAR, en el último se demuestra una causalidad bidireccional entre las variables.

Finalmente, en cuanto a las variables de control capital físico y trabajo, varias investigaciones demuestran la importancia de estos dos factores en el desempeño económico. Nakamura, Kaihatsu y Yagi (2018) en su investigación encuentran que la productividad de economía ha experimentado una desaceleración en los últimos años debido a la mala utilización de los factores como el capital físico y el capital humano. Por lo cual la mejora de la productividad laboral (que asocia estos dos factores) es una salida al crecimiento económico (Kameda, 2009). En este sentido, autores como Abubakar, Kassim y Yusoff (2015) mencionan que la formación de capital que se otorga a través del crecimiento financiero, contribuye a la mejora de las condiciones de las empresas, también asociado a la acumulación de capital humano, lo que a su vez se traduce 
en crecimiento económico. Alvarado, Iñiguez y Ponce (2017) indican que el capital físico como el trabajo son determinantes del crecimiento económico, sin embargo el capital físico es el factor fundamental del crecimiento económico, por encima del trabajo, en los países de ingresos altos de América Latina, en contraste con los países de ingreso medios altos y medio bajos, la fuerza laboral tiene mayor efecto en el crecimiento económico. Baudino (2016) también afirma que el impacto del capital físico es el componente más trascendental en el crecimiento económico, por encima de la mano de obra. Sin embargo, pese a que el capital físico es el factor más preciso para el crecimiento económico, este debe estar asociado con el factor trabajo (Pablo-Romero y Gómez-Calero, 2013; Voudouris, Ayres, Serrenho y Kiose, 2015). En esta misma línea, Ahmed, Mahalik y Shahbaz (2016) mencionan que el capital está asociado positiva y estadísticamente significativo con el crecimiento económico, a diferencia de la fuerza laboral que tiene un efecto positivo pero no significativo en el crecimiento económico.

Desde el punto de vista de la relación entre las variables macroeconómicas, resulta de interés la evaluación de la existencia de relaciones de equilibrio entre las variables, toda vez que los agregados macroeconómicos pueden responder en el corto y luego plazo de forma separada o conjunta. Los métodos más utilizados para evaluar las relaciones de equilibrio normalmente son los modelos de vectores autoregresivos y de corrección de error, debido a que estos modelos permiten capturar la dinámica temporal de la relación entre las variables. Siguiendo a Johansen (1991), la existencia de una relación de corto plazo implica que la variable dependiente responde de forma inmediata ante cambios en las variables independientes. En esta investigación en particular, el producto cambiará de forma inmediata si existe un cambio en la inversión extranjera directa y la libertad económica, mientras que el equilibrio de largo plazo implica que las variables se mueven en el largo plazo de forma conjunta y simultánea. La segunda parte de la siguiente sección plantea el mecanismo empleado para determinar la relación de corto y largo plazo entre las series.

\section{Materiales y Métodos}

\subsection{Datos}

La base de datos utilizada en la presente investigación fue obtenida del WDI del Banco Mundial (2017) y de la base de datos de Heritage Fundación (2017). Las variables son series temporales anuales correspondientes al periodo 1980-2017 para Ecuador. En las regresiones econométricas, la variable dependiente es el logaritmo del PIB per cápita a precios constantes del 2010 (PIBpc), y las variables independientes son los logaritmos del capital físico (K), la fuerza laboral (L), la entrada de capital neto (IED), la misma que fue deflactada para el posterior análisis, y finalmente la variable índice de libertad económica (LE) tomada de la base datos de Heritage Foundation (2017). La variable libertad económica se obtiene de la variable proxy libertad comercial, variable que es medida en un índice de 0 a 100, en donde 0 significa ausencia de libertad económica y 100 significa libertad económica total.

En el caso de Ecuador, las políticas para fomentar las entradas netas de capital no han sido claras. La Constitución Política de Ecuador establece que la IED será complementaria a la nacional y que se debe enfocar en la diversificación de las actividades productivas. De hecho, el Código Orgánico de la Producción y la Ley de Fomento productivo buscan reforzar las alianzas públicoprivadas para aumentar las entradas de inversión. Sin embargo, Alvarado, Iñiguez y Ponce (2017) señalan que la IED en Ecuador se concentra en la extracción de materias primas y en menor medida en algunas actividades orientadas al sector primario-exportador y telecomunicaciones. 
Tabla 1. Definición de las variables

\begin{tabular}{|c|c|c|c|}
\hline Variable & Abreviación & Descripción & Escala \\
\hline \multicolumn{4}{|l|}{ Dependiente } \\
\hline PIB per cápita & $\mathrm{PIBpc}$ & $\begin{array}{l}\text { Cociente entre el Producto Interno Bruto en dólares y la pobla- } \\
\text { ción total. }\end{array}$ & logaritmo \\
\hline \multicolumn{4}{|l|}{ Controles } \\
\hline Capital físico & K & Formación bruta de capital en dólares constantes del año 2010 & logaritmo \\
\hline Fuerza Laboral & L & $\begin{array}{l}\text { Tasa de participación en la fuerza laboral, total (\% del total de } \\
\text { la población entre 15-64 años) }\end{array}$ & logaritmo \\
\hline \multicolumn{4}{|l|}{ Independientes } \\
\hline Inversión extranjera & IED & Entrada neta de capital en dólares. & logaritmo \\
\hline \multirow[t]{2}{*}{ Libertad económica } & \multirow[t]{2}{*}{ LE } & Índice de libertad comercial Índice de 0 a 100. & \multirow[t]{2}{*}{ logaritmo } \\
\hline & & 0 Indica menos libertad comercial y 100 más libertad comercial & \\
\hline
\end{tabular}

Fuente: Banco Mundial, Heritage foundation

Elaboración: Los editores

En el Gráfico 1 muestra el comportamiento de la inversión extranjera directa, libertad económica y crecimiento económico. En donde las variables advierten una tendencia creciente a lo largo de los años en la variable del PIBpc y un pico decreciente en la IED en los años 2000, fruto de la crisis económica que derivo en el cambio de moneda, en cuanto a la libertad económica se observa un pico decreciente en el año 2001, cuyo decrecimiento se debe a la crisis experimentada por Ecuador en este periodo. Así mismo se observa en la variable $\mathrm{k}$ un decrecimiento dentro del periodo antes mencionado, y por otro lado la fuerza laboral experimentó un significativo aumento a partir del año 1987. Dado el comportamiento tendencial de los paneles se procede a la aplicación del test de Dickey y Fuller (1979).

Gráfico 1. Evolución de las variables utilizadas en el modelo econométrico
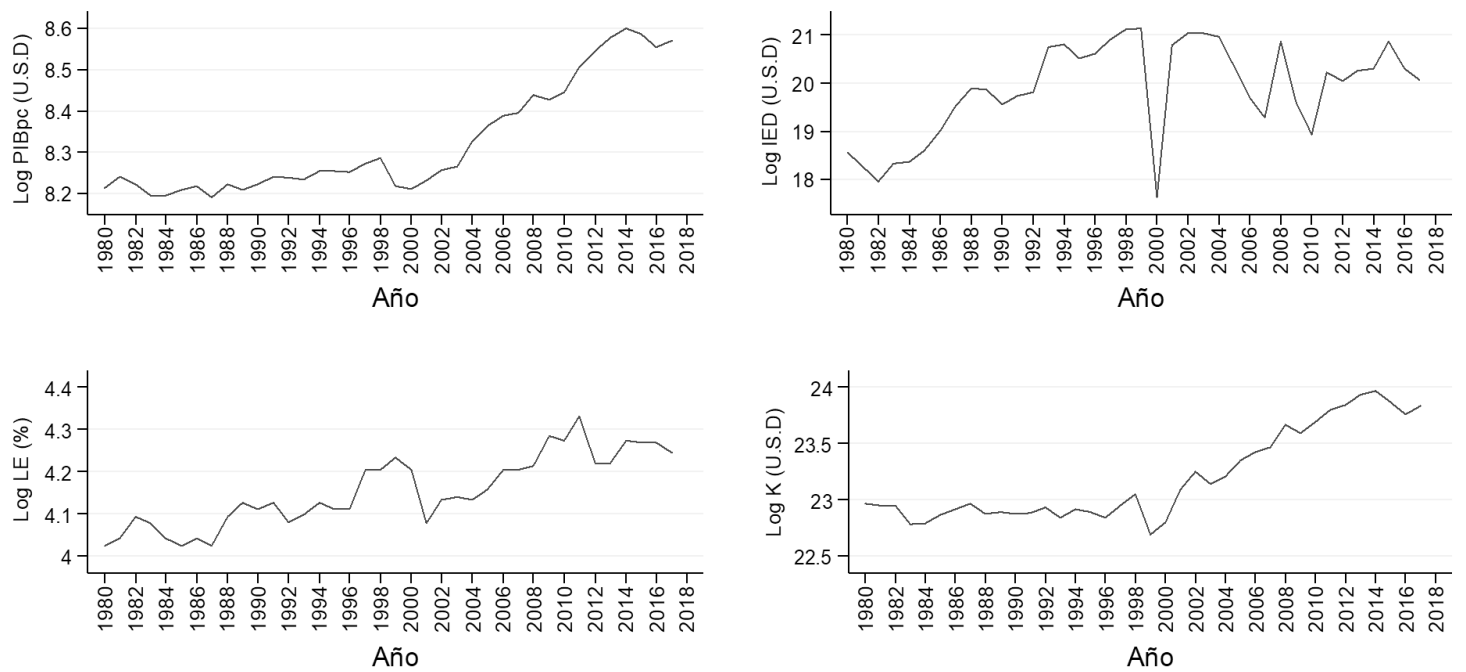


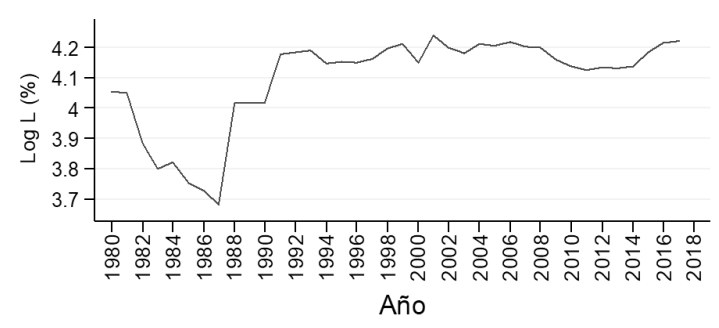

Fuente: Elaboración propia con datos del Banco Mundial y Heritage Fundation (2017).

Elaboración: Los autores.

En el Gráfico 2, se demuestra las variables en primera diferencia, en donde se puede observar que han perdido la característica tendencial y se vuelven series estacionarias, pues es necesario estacionarizar las series, esto lo comprueba más adelante el test de Dickey y Fuller (1979).

Gráfico 2. Evolución de las variables utilizadas en el modelo econométrico
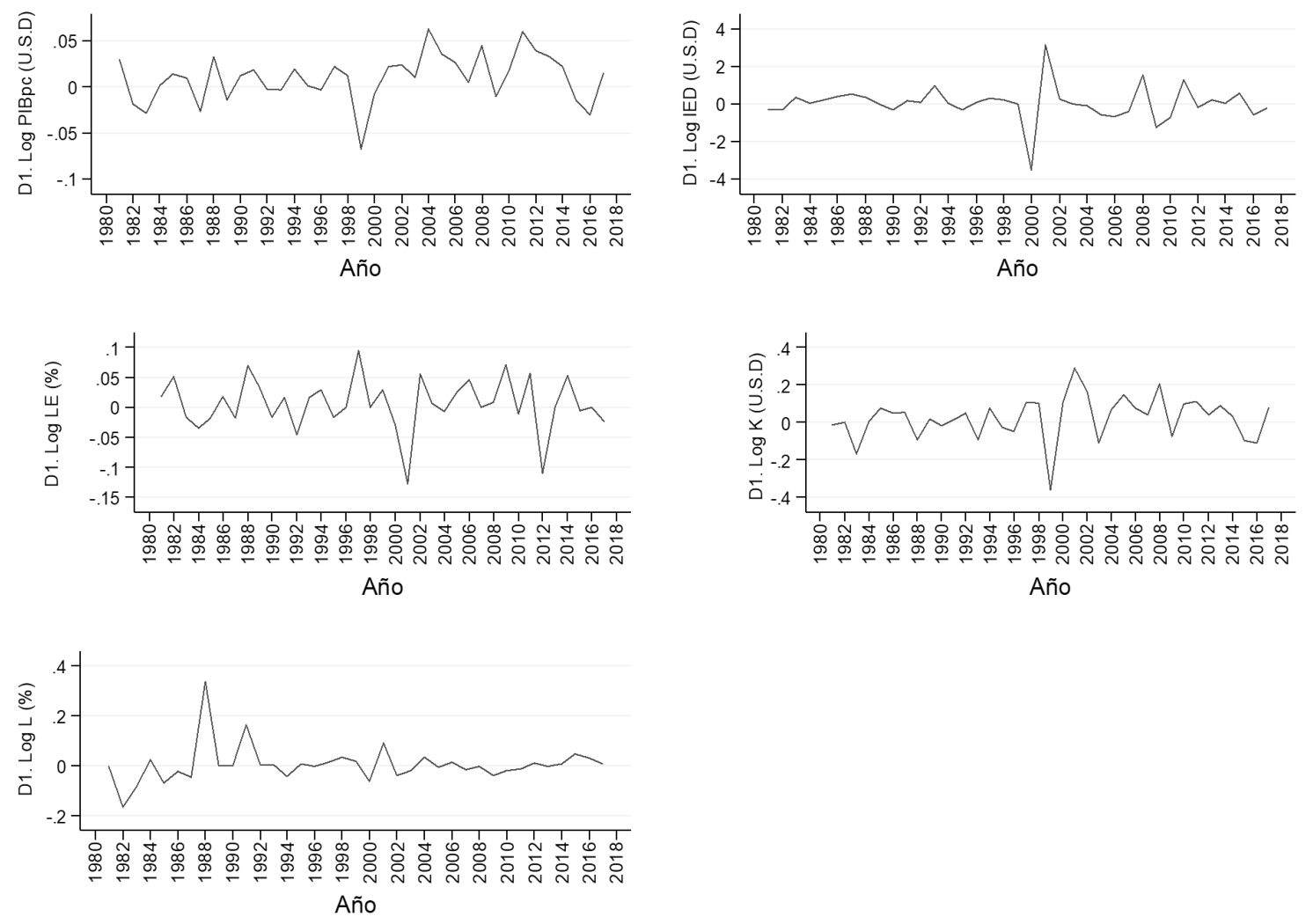

Fuente: Elaboración propia con datos del Banco Mundial y Heritage Fundation (2017) Elaboración: Los autores 


\subsection{Metodología}

Para determinar la existencia de relaciones de equilibrio de corto y largo plazo entre las variables, se procedió inicialmente a realizar el test de Dickey y Fuller (1979) para identificar la estacionariedad de las series de tiempo, en segundo lugar, se estimó modelos ECM y ARDL y por último para establecer relaciones de causalidad entre las variables, se aplicó la prueba de Engle y Granger (1987). El modelo de crecimiento de Solow se utiliza como marco teórico. El modelo muestra que la producción nacional $(\mathrm{Y})$ está determinada por tres factores: capital $(\mathrm{K})$, mano de obra $(\mathrm{L})$ y progreso técnico $(\mathrm{A})$. La función de producción toma la siguiente forma:

$$
\mathrm{Y}=\mathrm{Af}(\mathrm{K}, \mathrm{L})
$$

En la mayoría de los casos, el modelo de Solow se expresa como una función de producción de Cobb-Douglas que toma la siguiente forma:

$$
\mathrm{Y}=\mathrm{AK} \mathrm{K}^{\alpha} \mathrm{L}^{1-\alpha}
$$

Para hacer que la ecuación (2) sea lineal, aplicamos la función logarítmica e introducimos un término de error $\varepsilon$. Por lo tanto, la ecuación toma esta forma:

$$
\log (\mathrm{Y})=\log (\mathrm{A})+\alpha \log (\mathrm{K})+(1-\alpha) \log (\mathrm{L})+\varepsilon
$$

Suponiendo que el progreso técnico (A) sea un residuo. La ecuación (3) se estimará de la siguiente forma:

$$
\log (Y)=\beta_{0}+\beta_{1} \log (K)+\beta_{2} \log (L)+\varepsilon_{t}
$$

Donde $\beta \_0>0 ; \beta \_1>0 ; \beta \_2>0$ yY muestra el PIB per cápita. Introduciendo nuestras variables, el modelo se convierte en el siguiente:

$$
\log \text { IBPpc }_{t}=\beta_{0}+\beta_{1} \log \mathrm{K}_{\mathrm{t}}+\beta_{2} \log \mathrm{L}_{\mathrm{t}}+\beta_{2} \log \operatorname{IED}_{\mathrm{t}}+\beta_{2} \log _{\mathrm{LE}}+\beta_{2} \text { Dummy }_{\mathrm{t}}+\varepsilon_{\mathrm{t}}
$$

Donde $\operatorname{LogPIBpc} c_{t}$ representa el logaritmo del PIB per cápita en el período $t$; $\log \mathrm{K}_{t}$ representa el logaritmo de la formación bruta de capital fijo; Log $L_{t}$ representa el logaritmo de la fuerza laboral; LogIED representa el logaritmo de la inversión extranjera directa-entrada neta de capital, deflactada, Log Le $e_{t}$ representa la libertad económica y Dummy ${ }_{t}$ es una variable exógena que captura el efecto del cambio estructural de la economía como resultado de la crisis económica y financiera, y posterior dolarización de la economía ecuatoriana, este valor toma el valor de 0 antes del año 1999 y 1 desde el año 2000. Es importante destacar que son escasos las investigaciones que toman la libertad económica como una variable en un análisis.

La longitud del rezago del modelo fue determinada con el criterio de información de Akaike (1974), la cual sugiere la utilización de dos rezagos. Consecuentemente se considera el orden de integración de las variables mediante el test de Dickey y Fuller (1979) con el cual se determinó que todas las variables tienen un orden de integración I (1). La ecuación del modelo ARDL se escribe de la siguiente manera: 


$$
\begin{aligned}
& \triangle \operatorname{LogPIBpc}_{t}=\alpha_{0}+\alpha_{1} \log _{\operatorname{PIBpc}} \mathrm{c}_{t-i}+\alpha_{2} \log K_{t-i}+\alpha_{3} \log L_{t-i}+\alpha_{4} \log I E D_{t-i}+\alpha_{5} \log L E_{t-i} \\
& +\sum_{i=1}^{q} \rho_{1 i} \Delta \log P I B p c_{t-i}+\sum_{i=1}^{q} \rho_{2 i} \Delta \log K_{t-i}+\sum_{i=1}^{q} \rho_{3 i} \Delta \log L_{t-i}+\sum_{i=1}^{q} \rho_{4 i} \Delta \log I E D_{t-i} \\
& +\sum_{i=1}^{q} \rho_{5 i} \Delta \log L E_{t-i}+\varepsilon_{t}
\end{aligned}
$$

Donde " $\Delta$ " denota la primera diferencia de las variables. Para la estimación del modelo de corrección de errores (ECM), se analiza el término de error rezagado (ECT), el cual tiene que ser significativo para verificar la existencia de una relación de equilibrio a corto plazo entre las variables. La ecuación por estimar para el modelo ECM es la siguiente:

$$
\begin{aligned}
& \Delta \operatorname{LogPIBPc}_{t}=\alpha_{0}+\alpha_{1}{\log P I B P c_{t-i}}+\alpha_{2} \log K_{t-i}+\alpha_{3} \log L_{t-i}+\alpha_{4} \log I E D_{t-i}+\alpha_{5} \log L E_{t-i} \\
& +\sum_{i=1}^{q} \rho_{1 i} \Delta \operatorname{LogPIBPC}_{t-i}+\sum_{i=1}^{q} \rho_{2 i} \Delta \log K_{t-i}+\sum_{i=1}^{q} \rho_{3 i} \Delta \log L_{t-i}+\sum_{i=1}^{q} \rho_{4 i} \Delta \operatorname{LogIED_{t-i}} \\
& +\sum_{i=1}^{q} \rho_{5 i} \Delta \log L E_{t-i}+\alpha_{6} E C T_{t-1}+\gamma_{t}
\end{aligned}
$$

Para la prueba de causalidad de Engle y Granger (1987) se prueba que el evento X es visto como causando el evento Y, entonces el evento X debe preceder a Y (Hamilton, 1994, p.303). De esta forma Hamilton (1994) presenta para esta prueba una ecuación causal no restringida, de la siguiente manera

$$
Y_{t}=c_{1}+\sum_{i=1}^{p} a_{i} Y_{t-i}+\sum_{i=1}^{p} b_{i} X_{t-i}+\mu_{t}
$$

Y la luego ecuación restringida se expresa como:

$$
Y_{t}=c_{0}+\sum_{i=1}^{p} g_{i} Y_{t-i}+\mu_{t}
$$

El estadístico de prueba se calcula a partir de la suma de los residuos al cuadrado (RSS) de la ecuación no restringida (10) y la ecuación restringida (11) que solo es válida asintóticamente debido a la presencia de una variable dependiente retrasada en la regresión. De tal forma que el estadístico de la prueba se forma como:

$$
F=\frac{T\left(R S S_{0}-R S S_{1}\right)}{R S S_{1}}
$$

La cual sigue una distribución Chi-cuadrado. La prueba de causalidad de Granger está basada en la formalización del modelo de rezagos distribuidos con la finalidad de analizar relaciones causales bivariantes. Con el fin de asegurar que los resultados no estén sesgados por la sensibilidad del número de rezagos, esta prueba fue verificada luego de que el criterio de información de Akaike (1974) determina la longitud óptima de los rezagos. Este resultado fue contrastado con un criterio alternativo (Hannan and Quinn, 1979) y la longitud del rezago se mantuvo. Esta estrategia genera resultados estables y basados en un criterio estadístico en la inclusión del número de rezagos. Asimismo, es bien conocido que en 1999 la economía ecuatoriana tuvo un cambio estructural de la economía como resultado de la crisis económica y financiera y posterior dolarización de la economía. Este hecho fue capturado mediante la inclusión de una variable dummy, la cual toma valores de uno antes de la dolarización y cero luego de la dolarización. 
Tabla 2. Resumen de investigaciones que utilizan metodología similar a esta investigación

\begin{tabular}{|c|c|c|c|}
\hline Autor & Título & Año & Métodos \\
\hline Asali y Campoamor & $\begin{array}{l}\text { Sobre los efectos de la inversión extranjera } \\
\text { directa en la formación de capital humano }\end{array}$ & 2011 & Método de MCO. \\
\hline \multirow{2}{*}{$\begin{array}{l}\text { Álvarez, Herranz, Santiago, } \\
\text { Barraza, y Legato }\end{array}$} & \multirow{2}{*}{$\begin{array}{l}\text { Inversión Extranjera Directa y Crecimiento } \\
\text { Económico en Latinoamérica }\end{array}$} & \multirow[t]{2}{*}{2009} & Análisis de datos panel \\
\hline & & & $\begin{array}{l}\text { Método de Mínimos } \\
\text { Cuadrados en dos eta- } \\
\text { pas (Two-Stage EGLS ). }\end{array}$ \\
\hline Tafirenyika Sunde & $\begin{array}{l}\text { Foreign direct investment and economic } \\
\text { growth: ADRL and causality analysis for } \\
\text { South Africa }\end{array}$ & 2017 & $\begin{array}{l}\text { Modelos ARDL y ECMM, } \\
\text { análisis de causalidad de } \\
\text { Granger. }\end{array}$ \\
\hline \multirow[t]{2}{*}{ Iamsiraroj y Ulubaşoğlu } & $\begin{array}{l}\text { Foreign direct investment and economic } \\
\text { growth: A real relationship or }\end{array}$ & \multirow[t]{2}{*}{2015} & \multirow[t]{2}{*}{ Método de MCO. } \\
\hline & wishful thinking? & & \\
\hline \multirow[t]{2}{*}{ Abdouli y Sami } & $\begin{array}{l}\text { Investigating the causality links between } \\
\text { environmental quality, }\end{array}$ & \multirow[t]{2}{*}{2017} & \multirow{2}{*}{$\begin{array}{l}\text { Modelos VAR con datos } \\
\text { de panel y análisis de } \\
\text { causalidad. }\end{array}$} \\
\hline & $\begin{array}{l}\text { foreign direct investment and economic } \\
\text { growth in MENA countries. }\end{array}$ & & \\
\hline Evans y Opoku & $\begin{array}{l}\text { Foreign direct investment, regulations and } \\
\text { growth in sub-Saharan Africa }\end{array}$ & 2015 & $\begin{array}{l}\text { Método de estimación } \\
\text { GMM. }\end{array}$ \\
\hline lamsiraroj & $\begin{array}{l}\text { The foreign direct investment-economic } \\
\text { growth nexus }\end{array}$ & 2016 & Análisis de causalidad. \\
\hline \multirow[t]{2}{*}{ Dritsakia,y Stiakakisb } & $\begin{array}{l}\text { Foreign Direct Investments, Exports, and } \\
\text { Economic Growth }\end{array}$ & \multirow[t]{2}{*}{2014} & \multirow{2}{*}{$\begin{array}{l}\text { Modelos ARDL y el mo- } \\
\text { delo ECM-ARDL. Análisis } \\
\text { de causalidad. }\end{array}$} \\
\hline & in Croatia: A Time Series Analysis & & \\
\hline
\end{tabular}

Fuente: Science Direct.

Elaboración: Los editores.

\section{Discusión de resultados}

El objetivo de esta investigación es examinar el efecto de la IED y la LE sobre el PIB per cápita utilizando modelos de cointegración de corto y largo plazo. Antes de realizar el análisis, se debe plantear una posible limitación de esta investigación, dado que los datos utilizados en esta investigación son series agregadas anuales y el periodo de tiempo comprende 37 años, siendo difícil la obtención de series de datos anteriores al año 1980 para la variable libertad económica o en su defecto series trimestrales que serían series más óptimas para diferenciar relaciones de dependencia de corto y largo plazo. Aunque los modelos ADRL propuestos por Pesaran, Shin y Smith (PSS, 2001) pueden ser útiles aplicándolos en muestras pequeñas se debería tomar en cuenta para futuras investigaciones las limitaciones de los datos.

Primeramente, en esta sección se muestran los resultados del test de Dickey y Fuller (1979). El efecto tendencial se elimina al obtener la primera diferencia de las variables y las pruebas de raíces unitarias confirman que las variables tienen un orden de integración I (1). Una vez que se generó las primeras diferencias, las variables pasaron de tener un comportamiento tendencial a cíclico. Los resultados obtenidos se aprecian en la Tabla 3. 
Tabla 3. Resultados del test de ADF

\begin{tabular}{|c|c|c|c|c|c|c|c|c|c|}
\hline & \multicolumn{5}{|c|}{ Niveles } & \multicolumn{3}{|c|}{ 1era diferencia } & \multirow[t]{3}{*}{ I(q) } \\
\hline & \multirow[t]{2}{*}{ Valor calculado } & \multicolumn{3}{|c|}{ Valor critico } & \multirow{2}{*}{$\begin{array}{l}\text { Valor cal- } \\
\text { culado }\end{array}$} & \multicolumn{3}{|c|}{ Valor cítrico } & \\
\hline & & $1 \%$ & $5 \%$ & $10 \%$ & & $1 \%$ & $5 \%$ & $10 \%$ & \\
\hline $\log$ PIBpc $_{t}$ & 0,69 & $-3,68$ & $-2,97$ & $-2,62$ & $-5,28$ & $-3,69$ & $-2,98$ & $-2,62$ & 1 \\
\hline $\log K_{t}$ & 0,43 & $-3,44$ & $-2,26$ & $-2,54$ & $-6,33$ & $-3,22$ & $-2,32$ & $-2,22$ & 1 \\
\hline $\log L_{t}$ & $-1,34$ & $-3,33$ & $-2,32$ & $-2,12$ & $-6,09$ & $-3,7$ & $-2,58$ & $-2,54$ & 1 \\
\hline $\log \operatorname{IED}_{t}$ & $-2,41$ & $-3,7$ & $-2,58$ & $-2,37$ & $-6,54$ & $-3,71$ & $-2,98$ & $-2,62$ & 1 \\
\hline $\log L E_{t}$ & $-1,42$ & $-3,68$ & $-2,97$ & $\mid-2,62$ & $-6,43$ & $-3,7$ & $-2,98$ & $-2,62$ & 1 \\
\hline
\end{tabular}

Fuente: Banco Mundial, Heritage foundation

Elaboración: Los editores

Posterior a ello, se realizó el modelo ARDL para verificar la relación de cointegración de largo plazo entre las variables de la función. Para ello es necesario utilizar el test PSS que reporta valores críticos para muestras mayores a 30 observaciones El estadístico $F(15,86)$ supera los valores críticos como lo indica la Tabla 4, por lo tanto, se indica la existencia de una relación de largo plazo entre las variables.

Tabla 4. Resultados del Modelo ARDL

\begin{tabular}{|l|l|l|l|l|l|l|l|l|}
\hline \multicolumn{1}{|c|}{} & \multicolumn{9}{c|}{ valores críticos } \\
\hline \multicolumn{1}{|c|}{ k_4 } & \multicolumn{3}{|c|}{$\mathbf{1 0 \%}$} & \multicolumn{2}{c|}{$\mathbf{5 \%}$} & \multicolumn{2}{c|}{$\mathbf{2 , 5 0 \%}$} & \multicolumn{2}{c|}{$\mathbf{1 \%}$} \\
\hline & (I_0) & (I_1) & (I_0) & (I_1) & (I_0) & (I_1) & (I_0) & (I_1) \\
\hline Prueba F & L_1 & L_1 & L_5 & L_5 & L_025 & L_025 & L_01 & L_01 \\
\hline 15,86 & 2,45 & 3,52 & 2,79 & 4,85 & 3,41 & 4,52 & 3,15 & 5,36 \\
\hline
\end{tabular}

Fuente: Banco Mundial, Heritage foundation.

Elaboración: Los editores

Los resultados de este estudio son coincidentes con los encontrados por Sunde (2017); Fadhil y Almsafir (2015) y Pegkas (2015) quiénes encontraron una relación a largo plazo entre el crecimiento económico y la IED. Los resultados también coinciden con los estudios de Sun (2011) demostrando que existe una relación de cointegración de largo plazo entre la LE y la IED. En la investigación se procede además la estimación del modelo de ECM con el fin de verificar la existencia de un equilibrio de corto plazo. El resultado del test sugiere la existencia de dicho equilibrio, lo que implica que el crecimiento económico es sensible a los cambios en la inversión y libertad económica en el corto plazo. 
Tabla 5. Resultados del modelo de Corrección de error ECM

\begin{tabular}{|c|c|c|c|c|c|c|}
\hline Beta & Coef. & Std. Err. & $z$ & $P>Z$ & [95\% Conf. & Interval] \\
\hline$\Delta \log \mathrm{PIB}_{\mathrm{t}}$ & 1 & . & . & . & . & . \\
\hline$\Delta \log \mathrm{K}_{\mathrm{t}}$ & $-1,23 E-8$ & 0,431 & 0 & 1 & $-0,844$ & 0,844 \\
\hline$\Delta \log \mathrm{L}_{\mathrm{t}}$ & $-1,48 E-9$ & 0,644 & 0 & 1 & $-1,262$ & 1,262 \\
\hline$\Delta \log \operatorname{IED}_{t}$ & 0,144 & 0,055 & 2,64 & 0,008 & 0,037 & 0,251 \\
\hline$\Delta \log \mathrm{LE}_{\mathrm{t}}$ & 0,080 & 1,161 & 0,07 & 0,945 & $-2,195$ & 2,354 \\
\hline Dummy $_{t}$ & -.0166789 & .3774074 & -0.04 & 0.965 & -.7563838 & .723026 \\
\hline $\mathrm{ECT}_{\mathrm{t}-1}$ & & 0,377 & $-2,65$ & 0,008 & $-1,738$ & $-0,262$ \\
\hline _cons & $-0,020$ & . & . & . & . & . \\
\hline
\end{tabular}

Fuente: Banco Mundial, Heritage foundation.

Elaboración: Los editores.

Estos resultados son contrarios a los encontrados por Basu y Guariglia (2007), quienes llegan a la conclusión de que puede que el capital extranjero no sea necesariamente mejor para la difusión de la tecnología y el crecimiento en el corto plazo, por ejemplo en el caso de países con alta deuda externa. Luego mediante las pruebas de causalidad de Granger probamos la dirección de la causalidad de las variables, la Tabla 6 reporta estos resultados.

Tabla 6. Resultados de las pruebas de causalidad de Granger

\begin{tabular}{|c|c|c|}
\hline Causalidad & Prob > chi2 & chi2(2) \\
\hline$\Delta \log \mathrm{PIBpc} \rightarrow \Delta \log \mathrm{K}$ & 0,82 & 0,4 \\
\hline$\Delta \log \mathrm{K} \rightarrow \Delta \log \mathrm{PIBpc}$ & 0,37 & 1,97 \\
\hline$\Delta \log$ PIBpc $\rightarrow \Delta \log \mathrm{L}$ & 0,39 & 1,85 \\
\hline$\Delta \log \mathrm{L} \rightarrow \Delta \log \mathrm{PIBpc}$ & 0,62 & 1,95 \\
\hline$\Delta \log$ PIBpc $\rightarrow \Delta \log$ IED & 0,28 & 2,51 \\
\hline$\Delta \log$ IED $\rightarrow \Delta \log$ PIBpc & 0,01 & 8,07 \\
\hline$\Delta \log \mathrm{PIBpc} \rightarrow \Delta \log \mathrm{LE}$ & 0,44 & 1,62 \\
\hline$\Delta \log \mathrm{LE} \rightarrow \Delta \log$ PIBpc & 0,22 & 2,65 \\
\hline$\Delta \log \mathrm{K} \rightarrow \Delta \log \mathrm{L}$ & 0,45 & 1,59 \\
\hline$\Delta \log \mathrm{L} \rightarrow \Delta \log \mathrm{K}$ & 0,83 & 0,37 \\
\hline$\Delta \log \mathrm{K} \rightarrow \Delta \log$ IED & 0,04 & 6,17 \\
\hline$\Delta \log$ IED $\rightarrow \Delta \log \mathrm{K}$ & 0 & 16,01 \\
\hline$\Delta \log \mathrm{K} \rightarrow \Delta \log \mathrm{LE}$ & 0,15 & 3,77 \\
\hline$\Delta \log \mathrm{LE} \rightarrow \Delta \log \mathrm{K}$ & 0,1 & 4,54 \\
\hline$\Delta \log \mathrm{L} \rightarrow \Delta \log$ IED & 0,68 & 0,75 \\
\hline$\Delta \log$ IED $\rightarrow \Delta \log \mathrm{L}$ & 0,87 & 0,27 \\
\hline$\Delta \log \mathrm{L} \rightarrow \Delta \log \mathrm{LE}$ & 0,48 & 1,44 \\
\hline$\Delta \log \mathrm{LE} \rightarrow \Delta \log \mathrm{L}$ & 0,68 & 0,76 \\
\hline
\end{tabular}




\begin{tabular}{|l|l|l|}
\hline$\Delta$ Log IED $\rightarrow \Delta \log \mathrm{LE}$ & 0,16 & 3,68 \\
\hline$\Delta \log \mathrm{LE} \rightarrow \Delta \log$ IED & 0,06 & 5,43 \\
\hline
\end{tabular}

Fuente: Banco Mundial, Heritage foundation.

\section{Elaboración: Los editores.}

Finalmente, el test de causalidad de Granger determinó la existencia de causalidad bidireccional entre la formación bruta de capital fijo y la IED. También se encontró causalidad unidireccional desde la IED hacia el PIBpc. Estos últimos hallazgos son contrario a los hallazgos de Abdouli y Hammami (2017) y Dritsaki y Stiakakis (2014), quienes demuestran causalidad desde el crecimiento económico hacia la inversión extranjera directa. De la misma manera Gunby, Jin y Robert Reed (2017) que afirma que el fomento de la IED no siempre mejora la economía, y las relaciones de causalidad pueden darse de manera inversa $\left(\mathrm{PIB}_{\mathrm{t}} \rightarrow \mathrm{IED}_{\mathrm{t}}\right)$ incluso al trasplantar las políticas que favorecen a unos países, probablemente tendrán poco o nulo impacto en otros. En el mismo contexto Szkorupová (2014) asevera que existen variables que poseen un mayor efecto en el PIB un ejemplo de esto son las exportaciones.

En investigaciones similares Basu y Guariglia (2007) y Sun (2011) muestran que el crecimiento económico conduciría inevitablemente al aumento de la IED y viceversa. Se afirma además que la inversión promueve tanto la desigualdad como el crecimiento, es decir podría exacerbar la desigualdad, especialmente en un entorno en el que los pobres no pueden acceder a la tecnología moderna. Países con mayor libertad económica logran un ingreso per cápita y una tasa de crecimiento sustancialmente más altos ya que proporcionan incentivos pero muchas veces se ve restringida por altos impuestos y regulaciones; por la inseguridad de los derechos de propiedad; por restricciones al intercambio tanto nacional como internacional; por costosas regulaciones al crédito, mano de obra y empresas, como también por devaluaciones de la moneda.

Para el caso de Ecuador la inversión y la libertad económica son importantes tanto en el largo como en el corto plazo (Haan y Sturm, 2000; Compton, Giedeman y Hoover, 2011; Moussa, Çaha y Karagöz, 2016; Islam, 2018) y expresan la influencia y contribución especialmente de esta última, aunque no se descarta la presencia de otros factores que pueden tener mayor ponderación o encontrarse más estrechamente relacionadas con el crecimiento económico como lo pueden ser la tecnología (Khatun y Ahamad, 2015), la inflación e impuestos, que aparecen como un indicadores de estabilidad económica, según Demirhan y Masca, (2008) bajas tasas de inflación e impositivas han sido efectivas para atraer IED en países en desarrollo, o también las exportaciones de servicios que poseen causalidad unidireccional con la IED (Dash y Parida, 2013). Cabe recalcar que la calidad de la inversión extranjera es clave, dado que los encadenamientos productivos son importantes, al igual que la apertura comercial en condiciones óptima para el país generará un crecimiento sustentable.

\section{Conclusiones e implicaciones de política}

La investigación analiza el efecto de la inversión extranjera directa y la libertad económica en el crecimiento económico del Ecuador utilizando datos de series de tiempo en el periodo 1980-2017. Se encontró una relación de equilibrio de corto plazo, y largo plazo entre las variables, existiendo además causalidad bidireccional entre la formación bruta de capital fijo y la IED y causalidad unidireccional desde la IED hacia el PIB. Concordando con Basu y Guariglia (2007), mayores niveles de inversión tienden a lograr una mayor eficiencia de factores y consecuentemente tasas de crecimiento más altas. 
Dentro de las implicaciones de política, siguiendo a Solow (1956), para lograr un crecimiento económico, es importante alcanzar el pleno empleo, una alternativa es el aumento del gasto público. Estudios realizados por Carkovic y Levine (2002) y Basu y Guariglia (2007) proponen subsidios no solo para atraer capital extranjero, sino también subsidios educativos que podrían ayudar a los pobres a alcanzar la cantidad mínima de capital humano necesario para convertirse en emprendedores con pequeños negocios que ayuden a dinamizar las economías locales a largo plazo, mientras que Murphy y Topel (2016) apuntan a proponer remedios para el problema de la desigualdad que se encuentran en el lado de la oferta, específicamente en las políticas que fomentan o permiten la adquisición de habilidades o fomentan la inmigración de personas altamente calificadas. Finalmente Yao (2006) sugiere políticas de desarrollo para economías en crecimiento y en transición: promoción y adopción de exportaciones de la tecnología mundial y las prácticas comerciales.

\section{Agradecimiento}

Los autores expresan su gratitud con el Club de Investigación de Economía-CIE, Loja Ecuador. 


\section{Referencias bibliográficas:}

- Abdouli, M., y Hammami, S. (2017). Investigating the causality links between environmental quality, foreign direct investment and economic growth in MENA countries. International Business Review, 26(2), 264-278. https://doi.org/10.1016/j.ibusrev.2016.07.004

- Abubakar, A., Kassim, S. H., y Yusoff, M. B. (2015). Financial Development, Human Capital Accumulation and Economic Growth: Empirical Evidence from the Economic Community of West African States (ECOWAS). Procedia - Social and Behavioral Sciences, 172, 96-103. http://doi.org/10.1016/j.sbspro.2015.01.341

- Adams, S., y Opoku, E. E. O. (2015). Foreign direct investment, regulations and growth in sub-Saharan Africa. Economic Analysis and Policy, 47, 48-56. https://doi.org/10.1016/j. eap.2015.07.001

- Ahmed, K., Mahalik, M. K., y Shahbaz, M. (2016). Dynamics between economic growth, labor, capital and natural resource abundance in Iran: An application of the combined cointegration approach. Resources Policy, 49, 213-221. http://doi.org/10.1016/j.resourpol.2016.06.005

- Alfaro, L., Chanda, A., Kalemli-Ozcan, S., y Sayek, S. (2004). FDI and economic growth: The role of local financial markets. Journal of International Economics, 64(1), 89-112. http://doi. org/10.1016/S0022-1996(03)00081-3

- Almfraji, M. A., y Almsafir, M. K. (2014). Foreign Direct Investment and Economic Growth Literature Review from 1994 to 2012. Procedia - Social and Behavioral Sciences, 129, 206213. http://doi.org/10.1016/j.sbspro.2014.03.668

- Alvarado, R., Iñiguez, M., y Ponce, P. (2017). Foreign direct investment and economic growth in Latin America. Economic Analysis and Policy, 56, 176-187. http://doi.org/10.1016/j. eap.2017.09.006

- Akaike, H. (1974), A New Look at the Statistical Model Identification, ieee Transactions On Automatic Control, vol. 19, núm. 6.

- Anwar, S., y Nguyen, L. P. (2010). Foreign direct investment and economic growth in Vietnam. Asia Pacific Business Review, 16(1-2), 183-202. https://doi.org/10.1080/10438590802511031

- Anwar, S., y Sun, S. (2011). Financial development, foreign investment and economic growth in Malaysia. Journal of Asian Economics, 22(4), 335-342. https://doi.org/10.1016/j. asieco.2011.04.001

- Azman-Saini, W. N. W., Baharumshah, A. Z., y Law, S. H. (2010). Foreign direct investment, economic freedom and economic growth: International evidence. Economic Modelling, 27(5), 1079-1089. https://doi.org/10.1016/j.econmod.2010.04.001

- Banco Mundial (2017). World Development Indicators.Basu, P., y Guariglia, A. (2007). Foreign Direct Investment, inequality, and growth. Journal of Macroeconomics, 29(4), 824-839. https:// doi.org/10.1016/j.jmacro.2006.02.004

- Baudino, M. (2016). The impact of human and physical capital accumulation on Chinese growth after 1994: A spatial econometric approach. World Development Perspectives, 2, 11-16. http:// doi.org/10.1016/j.wdp.2016.08.001

- Bénassy-Quéré, A., Fontagné, L., y Lahrèche-Révil, A. (2001). Exchange-rate strategies in the competition for attracting foreign direct investment. Journal of the Japanese and international Economies, 15(2), 178-198. 
- Belaşcu, L., Popovici, O., y Horobeț, A. (2018). Foreign Direct Investments and Economic Growth in Central and Eastern Europe: A Panel-Based Analysis. In Emerging Issues in the Global Economy (pp. 35-46). Springer, Cham.

- Belloumi, M. (2014). The relationship between trade, FDI and economic growth in Tunisia: An application of the autoregressive distributed lag model. Economic Systems, 38(2), 269-287. http://doi.org/10.1016/j.ecosys.2013.09.002

- Bengoa, M., y Sanchez-Robles, B. (2003). Foreign direct investment, economic freedom and growth: New evidence from Latin America. European Journal of Political Economy, 19(3), 529545. https://doi.org/10.1016/S0176-2680(03)00011-9

- Bhagwati, J., y Srinivasan, T. N. (2002). Trade and poverty in the poor countries. American Economic Review, 92(2), 180-183.

- Borensztein, E., De Gregorio, J., y Lee, J. W. (1998). How does foreign direct investment affect economic growth? 1. Journal of international Economics, 45(1), 115-135

- Carkovic, M., y Levine, R. (2002). Does Foreign Direct Investment Accelerate Economic Growth?, 1-11. https://doi.org/10.2139/ssrn.314924

- Chen, J.-E., y Zulkifli, S. A. M. (2012). Malaysian Outward FDI and Economic Growth. Procedia - Social and Behavioral Sciences, 65(ICIBSoS), 717-722. http://doi.org/10.1016/j. sbspro.2012.11.189

- Compton, R. A., Giedeman, D. C., y Hoover, G. A. (2014). A distributional analysis of the benefits of economic freedom. European Journal of Political Economy, 33, 121-133. https:// doi.org/10.1016/j.ejpoleco.2013.12.001

- Dash, R. K., y Parida, P. C. (2013). FDI, services trade and economic growth in India: Empirical evidence on causal links. Empirical Economics, 45(1), 217-238. https://doi.org/10.1007/ s00181-012-0621-1

- Dawson, J. W. (2003). Causality in the freedom - Growth relationship. European Journal of Political Economy, 19(3), 479-495. https://doi.org/10.1016/S0176-2680(03)00009-0

- De Haan, J., y Sturm, J.-E. (2000). On the relationship between economic freedom and economic growth. European Journal of Political Economy, 16(2), 215-241. https://doi. org/10.1016/S0176-2680(99)00065-8

- Demirhan, E., y Masca, M. (2008). Determinants of foreign direct investment flows to developing countries: a cross-sectional analysis. Prague Economic Papers, 17(4), 356-369. https://doi. org/10.18267/j.pep.337

- Dickey, D. A., y Fuller, W. A. (1979). Distribution of the estimators for autoregressive time series with a unit root. Journal of the American statistical association, 74(366a), 427-431.

- Dritsaki, C., y Stiakakis, E. (2014). Foreign Direct Investments, Exports, and Economic Growth in Croatia: A Time Series Analysis. Procedia Economics and Finance, 14(1992), 181-190. https://doi.org/10.1016/S2212-5671(14)00701-1.

- Engle, R.F., y Granger, C.W.J., 1987. Co-integration and error-correction: Representation, estimation and testing. Econometrica 55, 252-276.

- Fadhil, M. A., y Almsafir, M. K. (2015). The Role of FDI Inflows in Economic Growth in Malaysia (Time Series: 1975-2010). Procedia Economics and Finance, 23(October 2014), 1558-1566. http://doi.org/10.1016/S2212-5671(15)00498-0 
- Friedman, J. (Ed.). (1996). The rational choice controversy: Economic models of politics reconsidered (No. 1-2). Yale University Press.

- Gomes Neto, D., y Veiga, F. J. (2013). Financial globalization, convergence and growth: The role of foreign direct investment. Journal of International Money and Finance, 37, 161-186. https://doi.org/10.1016/j.jimonfin.2013.04.005

- Gunby, P., Jin, Y., y Robert Reed, W. (2017). Did FDI Really Cause Chinese Economic Growth? A Meta-Analysis. World Development, 90, 242-255. http://doi.org/10.1016/j.worlddev.2016.10.001

- Hamilton, J. D. (1994). Time series analysis (Vol. 2, pp. 690-696). Princeton, NJ: Princeton university press.

- Hannan, E. J., y Quinn, B. G. (1979). The determination of the order of an autoregression. Journal of the Royal Statistical Society. Series B (Methodological), 190-195.

- Hayek, F. A. (2014). The constitution of liberty. Routledge.

- Heritage Fundación (2017). Index of Economic Freedom.

- Herranz, A. Á., y Barraza, J. S. E. (2009). Inversión Extranjera Directa y Crecimiento Económico en Latinoamérica, 20(6), 115-124. https://doi.org/10.1612/inf.tecnol.4116it.08

- Herrera-Echeverri, H., Haar, J., y Estévez-Bretón, J. B. (2014). Foreign direct investment, institutional quality, economic freedom and entrepreneurship in emerging markets. Journal of Business Research, 67(9), 1921-1932. https://doi.org/10.1016/j.jbusres.2013.11.020

- Huang, J., Chen, X., Huang, B., y Yang, X. (2016). Economic and environmental impacts of foreign direct investment in China: A spatial spillover analysis. China Economic Review. https:// doi.org/10.1016/j.chieco.2016.03.006

- Hussain, A. (2011). Foreign Direct Investment ( Fdi ) and Its Impact on the Productivity of Domestic, 792-812.

- Islam, M. R. (2018). Wealth inequality, democracy and economic freedom. Journal of Comparative Economics. https://doi.org/10.1016/j.jce.2018.01.002

- lamsiraroj, S., y Ulubaşoğlu, M. A. (2015). Foreign direct investment and economic growth: A real relationship or wishful thinking?. Economic Modelling, 51, 200-213

- lamsiraroj, S. (2016). The foreign direct investment-economic growth nexus. International Review of Economics and Finance, 42, 116-133. http://doi.org/10.1016/j.iref.2015.10.044

- Kameda, S. (2009), "Issues on Japan's Productivity: How Do We Evaluate Productivity Developments Since 2000?" Bank of Japan Working Paper Series, No. 09-J-11

- Khatun, F., y Ahamad, M. (2015). Foreign direct investment in the energy and power sector in Bangladesh: Implications for economic growth. Renewable and Sustainable Energy Reviews, 52, 1369-1377. https://doi.org/10.1016/j.rser.2015.08.017

- Miller, T., y Kim, A. (2016). Index of economic freedom: Promoting economic prosperity and freedom.

- Nakamura, K., Kaihatsu, S., y Yagi, T. (2018). Productivity improvement and economic growth: Lessons from Japan. Economic Analysis and Policy. http://doi.org/10.1016/j.eap.2018.11.002

- Nistor, P. (2014). FDI and Economic Growth, the Case of Romania. Procedia Economics and Finance, 15(14), 577-582. http://doi.org/10.1016/S2212-5671(14)00514-0 
- Leobardo, I., y Juárez, S. (2014). Libertad económica y crecimiento económico: teoría y evidencias. Recuperado de http://www.caminosdelalibertad.com/resources/uploads/ pdf/20130528_140321_4concursoensayo_1lugar.pdf

- Moussa, M., Çaha, H., y Karagöz, M. (2016). Review of Economic Freedom Impact on FDI: New Evidence from Fragile and Conflict Countries. Procedia Economics and Finance, 38(October 2015), 163-173. https://doi.org/10.1016/S2212-5671(16)30187-3

- Murphy, K. M., y Topel, R. H. (2016). Human Capital Investment, Inequality, and Economic Growth. Journal of Labor Economics, 34(S2), S99-S127. https://doi.org/10.1086/683779

- Oscar, H., Díaz, L., y Navarrete, R. A. (2017). Crecimiento , competitividad y restricción externa en América Latina, LXXVI, 53-80.

- Pablo-Romero, M. del P., y Gómez-Calero, M. de la P. (2013). A translog production function for the Spanish provinces: Impact of the human and physical capital in economic growth. Economic Modelling, 32(1), 77-87. http://doi.org/10.1016/j.econmod.2013.01.040

- Pesaran, M. H., Shin, Y., y Smith, R. J. (2001). Bounds testing approaches to the analysis of long run relationships. Journal of Applied Econometric, 16(3), 289-326. https://doi.org/10.1002/ jae.616

- Pegkas, P. (2015). The impact of FDI on economic growth in Eurozone countries. Journal of Economic Asymmetries, 12(2), 124-132. http://doi.org/10.1016/j.jeca.2015.05.001

- Ramírez, C. V. (2016). La Inversión Extranjera Directa. Saber, ciencia y libertad (Vol. 5).

- Sánchez, I. L. (2010). Libertad económica y crecimiento económico: teoría y evidencias. Memorias del Cuarto Concurso de Ensayo Caminos de la Libertad. México DF, México: Grupo Salinas.

- Solow, R. M. (1956). A Contribution to the Theory of Economic Growth. Source: The Quarterly Journal of Economics, 70(1), 65-94. https://doi.org/10.2307/1884513

- Sokhanvar, A. (2019). Does foreign direct investment accelerate tourism and economic growth within Europe? Tourism Management Perspectives, 29(September 2018), 86-96. http://doi. org/10.1016/j.tmp.2018.10.005

- Smith, A. (1776). The wealth of nations, book 1. London, Methuen y Co.

- Sturm, J. E. (2001). How robust is the relationship between economic freedom and economic growth? Applied Economics, 33(7), 839-844. https://doi.org/10.1080/00036840121977

- Sun, H. (2011). Co-Integration Study of Relationship between Foreign Direct Investment and Economic Growth. International Business Research, 4(4), 226-230. https://doi.org/10.5539/ ibr.v4n4p226

- Sunde, T. (2017). Foreign direct investment, exports and economic growth: ADRL and causality analysis for South Africa. Research in International Business and Finance, 41, 434-444. https://doi.org/10.1016/j.ribaf.2017.04.035

- Szkorupová, Z. (2014). A Causal Relationship between Foreign Direct Investment, Economic Growth and Export for Slovakia. Procedia Economics and Finance, 15(4), 123-128. https://doi. org/10.1016/S2212-5671(14)00458-4

- Tsai, P.-L. (1995). Foreign Direct Investment and Income Inequality: Further Evidence. World Development, 23(3), 469-483. https://doi.org/10.1016/0305-750X(95)00136-Z 
- Tung, S., y Cho, S. (2001). Determinants of Regional Investment Decisions in China: An Econometric Model of Tax Incentive Policy. Review of Quantitative Finance and Accounting, 17(1983), 167. https://doi.org/10.1023/A:1017925721627

- Voudouris, V., Ayres, R., Serrenho, A. C., y Kiose, D. (2015). The economic growth enigma revisited: The EU-15 since the 1970s. Energy Policy, 86, 812-832. http://doi.org/10.1016/j. enpol.2015.04.027

- Yao, S. (2006). On economic growth, FDI and exports in China. Applied Economics, 38(3), 339-351. https://doi.org/10.1080/00036840500368730 
FOREIGN DIRECT INVESTMENT AND ECONOMIC FREEDOM AS

Cristian Ortiz DETERMINANTS OF ECUADOR'S ECONOMIC GROWTH IN THE Andrea Salinas SHORT AND LONG TERM 